\title{
Proposed mechanism for anosmia during COVID-19: The role of local zinc distribution
}

\author{
Ozlem Equils ${ }^{*}$, Klaudi Lekaj ${ }^{1}$, Sahar Fattani ${ }^{1}$, Arthur $\mathrm{Wu}^{2}$ and Gene Liu ${ }^{2}$ \\ ${ }^{1} \mathrm{MiOra}$, public health education foundation (www.miora.org), Los Angeles, California, USA \\ ${ }^{2}$ Cedars-Sinai Medical Center, Division of Otolaryngology, Los Angeles, California, USA
}

The common cold is frequently associated with anosmia. A significant portion of COVID-19 patients has been reported to have anosmia and taste dysfunction [1]. A recent study from Germany found that among confirmed COVID-19 patients $47 \%$ had anosmia with a mean duration of anosmia of 8.9 days as well as an association with dysgeusia in $85 \%$ of cases [2].

There are multiple proposals for the pathogenesis of anosmia during COVID-19 including the direct and indirect toxic effect of SARSCoV-2 on neuronal cells [3] and toxicity to non-neuronal supportive cells such as olfactory epithelium sustentacular cells, microvillar cells and olfactory bulb pericytes $[4,5]$.

Here we propose that infections with coronaviruses including SARS-CoV-2 induce host immune responses in the nasopharyngeal mucosa; which may lead to local zinc deficiency and consequently transient anosmia and poor taste.

Zinc $(\mathrm{Zn})$ is an essential micronutrient and is the second most abundant metal in the human body, with 2 to 4 grams distributed throughout the whole body. Zinc is generally taken in through food or breast milk, is absorbed via several intestinal $\mathrm{Zn}$ transporters, and is released into the bloodstream. It is required for cell growth, differentiation, and survival and approximately $10 \%$ of the entire human genome can potentially bind $\mathrm{Zn}$ through $\mathrm{Zn}$-finger motifs [6]. Dietary $\mathrm{Zn}$ deficiency is common globally and causes thymic atrophy and depresses both innate and adaptive immune responses [7]. Zinc is a cofactor for proteins, it affects the structural and catalytic functions of enzymes and transcription factors and acts as a second messenger [8]. $\mathrm{Zn}$ homeostasis is tightly controlled by the coordinated activity of $\mathrm{Zn}$ transporters and metallothioneins and $\mathrm{Zn}$ itself behaves like a signaling molecule in response to extracellular stimuli.

Zinc deficiency is well known to cause anosmia and taste dysfunction. This is because one of the enzymes critical to maintain taste and smell function is a zinc dependent metalloenzyme called carbonic anhydrase (CA) [9]. Interestingly, different formulations of intranasal $\mathrm{Zn}$ have also been shown to cause anosmia, but the mechanisms for toxicity are complex, including oxidative stress, ATP depletion, cytoskeletal changes and apoptosis of olfactory neuronal cells, and is affected by many factors, such as concentration of zinc tested, the length of exposure, the cell type, and the presence of other toxic chemicals [10].

Myeloid and lymphoid cells manipulate intracellular and extracellular zinc metabolism via $\mathrm{Zn}$ binding proteins and transporters in response to immunological signals and infections [11]. Zinc is considered an acute phase reactant and $\mathrm{Zn}$ levels are redistributed during infection [12]. Systemic $\mathrm{Zn}$ deficiency is associated with decreased Th1 cytokines, interferon-gamma and interleukin (IL)-2, and unchanged production of Th2 cytokines (IL-4, IL-6, and IL-10); which causes a shift in Th1 to Th2 balance towards Th2 cytokine predominance [13].

Cellular $\mathrm{Zn}$ is important for viral replication, and chelation experiments have shown that decreases in $\mathrm{Zn}$ levels inhibit the replication of human immunodeficiency virus (HIV) and dengue fever virus [12]. Acute viral infection of the nasopharyngeal mucosa may lead to a decrease in local $\mathrm{Zn}$ level and this was proposed to be a part of normal host defense against respiratory pathogens [14].

Local shift in $\mathrm{Zn}$ homeostasis in the nose during common colds may lead to a local Th2 phenotype and may explain increased sneezing during colds. Sneezing is a protective reflex where air in the lungs is expelled out in reaction to an irritation in the nose. Sneezing may be an indicator of local $\mathrm{Zn}$ deficiency. Rhinovirus is the pathogen most frequently associated with common cold; once rhinovirus enters inside the nasal epithelial cell, there is no increase in the number of inflammatory cells, but neutrophils increase in the nasal mucosa and mucous secretions $[15,16]$. This may be due to Th 2 predominant immune responses such as kinins, leukotrienes, histamines, interleukin-1, interleukin-6 interleukin-8, tumor necrosis factor and RANTES (regulated by activation normal T-cell expressed and secreted); which can explain disease symptoms [17-19].

Early Th2 immune responses in the nasopharynx during viral upper respiratory tract infections may be protective. Th2 cytokines have also been shown to decrease the expression of SARS-CoV-2 receptor, angiotensin converting enzyme 2 (ACE-2) expression locally [20]. Reduced ACE-2 expression may be a part of host response to inhibit viral infection of neighboring cells in the nasopharynx.

Earlier studies with SARS-CoV have shown that in infected cells intracellular Zn may inhibit SARS-CoV RNA-dependent RNA polymerase (RdRp) elongation [21]. Chelation with magnesium EDTA reverses $\mathrm{Zn}$ effect on SARS-CoV RdRp. Zinc ions have also been shown to inhibit the proteolytic processing of replicase polyproteins in coronavirus infected cells [22].

${ }^{\star}$ Correspondence to: Ozlem Equils, MiOra, public health education foundation (www.miora.org), Los Angeles, California, USA, E-mail: ozlem@miora.org

Key words: COVID-19, anosmia, SARS, immune system, Th2, zinc

Received: May 19, 2020; Accepted: June 04, 2020; Published: June 08, 2020 
There is no data that show that $\mathrm{Zn}$ supplementation prevents viral infections, and the data on the effectiveness of $\mathrm{Zn}$ to treat common cold is heterogeneous [23,24]. We propose that an appropriate local host immune response to acute SARS-CoV-2 infection may include a change in nasopharyngeal $\mathrm{Zn}$ balance that leads to Th2 predominant immune response with reduced ACE- 2 expression, sneezing, anosmia and poor taste. Nasal mucosa is in continuum with the larger mucosal system (gastrointestinal (GI), respiratory, urinary, and genital tracts) [25]. In animal models, activation of nasal mucosa by viral antigens was shown to prime the immune environment in the lungs by increasing the infiltration with activated macrophages in the absence of direct pulmonary infection [25]. Anosmia in COVID-19 patients may be an indicator of COVID-19 prognosis. Yan $\mathrm{CH}$ and colleagues from University of California San Diego observed that patients with anosmia were less likely to require hospitalization and that COVID-19 resolved together with the resolution of anosmia [26]. Another study from Iran suggested that patients with anosmia were less likely to have fever, cough and dyspnea compared to those without anosmia $(87.9 \%$ vs $37.38 \%, 67.7 \%$ vs $18.98 \%$ and $18.6 \%$ vs $14.38 \%$ respectively) and hospitalization rate was low (1.1\%) among patients with anosmia [27].

Data from coronavirus seroconversion studies have also shown that nasal immunoglobulin responses are important in controlling the virus replication and disease severity [28]. Data is needed on the relationship between occurrence of anosmia and nasal antiSARS-CoV-2 immune responses as well as COVID-19 severity and prognosis. Better understanding of the mechanisms of anosmia may also help develop criteria for early anti-viral, convalescent plasma or antibody treatment.

\section{Funding}

This study was funded by MiOra public health education foundation (www.miora.org).

\section{Conflicts of interest}

There are no conflicts of interest.

\section{References}

1. Ahmad I, Rathore FA (2020) Neurological manifestations and complications of COVID-19: A Literature Review. Journal of Clinical Neuroscience. [Crossref]

2. Klopfenstein T, Toko L (2020) Features of anosmia in COVID-19. Médecine et Maladies infectieuses.

3. Li Z (2020) Neurological manifestations of patients with COVID-19: potential routes of SARS-CoV-2 neuroinvasion from the periphery to the brain. Frontiers of Medicine 1-9. [Crossref]

4. Vaira LA, Salzano G, Fois AG (2020) Potential pathogenesis of ageusia and anosmia in COVID-19 patients. In International forum of allergy \& rhinology.

5. Brann DH, Tsukahara T (2020) Non-neuronal expression of SARS-CoV-2 entry genes in the olfactory system suggests mechanisms underlying COVID-19-associated anosmia. bioRxiv. Neuroscience.

6. Andreini C (2006) Counting the zinc-proteins encoded in the human genome. Journal of Proteome Research 5: 196-201.
7. Shankar AH, Prasad AS (1998) Zinc and immune function: the biological basis of altered resistance to infection. The American Journal of Clinical Nutrition 68: $447 \mathrm{~S}-463 \mathrm{~S}$

8. Fukada T, Hojyo, Bin BH (2014) Zinc signal in growth control and bone diseases. In Zinc Signals in Cellular Functions and Disorders (pp. 249-267). Springer, Tokyo.

9. Komai M (2000) Zinc deficiency and taste dysfunction; Contribution of carbonic anhydrase, a zinc-metalloenzyme, to normal taste sensation. Biofactors 12: 65-70. [Crossref]

10. Hsieh H (2016) Mechanistic studies of the toxicity of zinc gluconate in the olfactory neuronal cell line Odora. Toxicology in Vitro 35: 24-30.

11. Vignesh KS, Deepe Jr GS (2016) Immunological orchestration of zinc homeostasis: the battle between host mechanisms and pathogen defenses. Archives of Biochemistry and Biophysics 611: 66-78.

12. Kar M, Khan NA (2019) Zinc chelation specifically inhibits early stages of Dengue virus replication by activation of NF-kappaB and induction of antiviral response in epithelial cells. Frontiers in immunology 10: 2347. [Crossref]

13. Prasad AS (2000) Effects of zinc deficiency on Th1 and Th2 cytokine shifts. The Journal of Infectious Diseases 182: S62-S68.

14. Wessels I, Maywald M, Rink L (2017) Zinc as a gatekeeper of immune function. Nutrients 9: 1286.

15. Fraenkel DJ, Bardin PG (1994) Immunohistochemical analysis of nasal biopsies during rhinovirus experimental colds. American Journal of Respiratory and Critical Care Medicine 150: 1130-1136. [Crossref]

16. Winther B (1994) Effects on the nasal mucosa of upper respiratory viruses (common cold). Danish Medical Bulletin 41: 193-204. [Crossref]

17. Noah TL (1995) Nasal cytokine production in viral acute upper respiratory infection of childhood. Journal of Infectious Diseases 171: 584-592.

18. Fritz RS (1999) Nasal cytokine and chemokine responses in experimental influenza A virus infection: results of a placebo-controlled trial of intravenous zanamivir treatment. The Journal of Infectious Diseases 180: 586-593.

19. Naclerio RM (1988). Kinins are generated during experimental rhinovirus colds Journal of Infectious Diseases 157: 133-142.

20. Jian L (2020). Perspective: COVID-19, Implications of nasal diseases and consequences for their management. Journal of Allergy and Clinical Immunology.

21. Te Velthuis AJ (2010) Zn2+ inhibits coronavirus and arterivirus RNA polymerase activity in vitro and zinc ionophores block the replication of these viruses in cell culture. PLoS Pathogens 6. [Crossref]

22. Denison MR (1992) Intracellular processing of the N-terminal ORF 1a proteins of the coronavirus MHV-A59 requires multiple proteolytic events. Virology 189: 274-284 [Crossref]

23. Singh M, Das RR (2013) Zinc for the common cold. Cochrane Database of Systematic Reviews 6.

24. Hemilä H (2017) Zinc lozenges and the common cold: a meta-analysis comparing zinc acetate and zinc gluconate, and the role of zinc dosage. JRSM Open 8: 2054270417694291. [Crossref]

25. Dwivedy A, Aich P (2011) Importance of innate mucosal immunity and the promises it holds. International Journal of General Medicine 4: 299.

26. Yan CH (2020, April) Association of chemosensory dysfunction and Covid-19 in patients presenting with influenza-like symptoms. In International Forum of Allergy \& Rhinology.

27. Bagheri SHR (2020) Coincidence of COVID-19 epidemic and olfactory dysfunction outbreak. Medrxiv.

28. Callow KA(1990) The time course of the immune response to experimental coronavirus infection of man. Epidemiology \& Infection 105: 435-446. [Crossref]

Copyright: (C2020 Equils O. This is an open-access article distributed under the terms of the Creative Commons Attribution License, which permits unrestricted use, distribution, and reproduction in any medium, provided the original author and source are credited. 